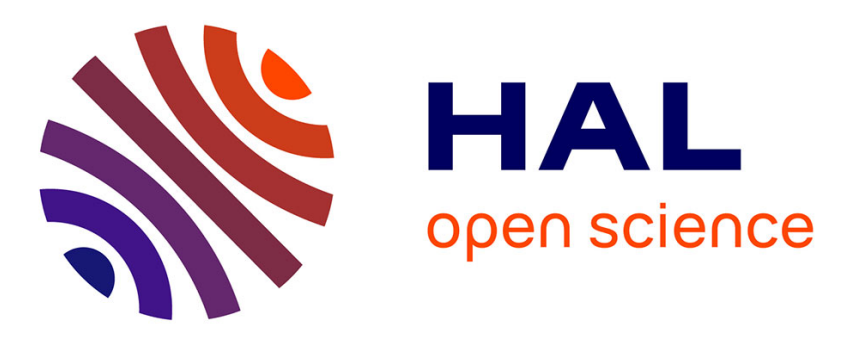

\title{
Hanbury-Brown Twiss noise correlation with time controlled quasi-particles in ballistic quantum conductors
}

\author{
Christian D.C. Glattli, P. Roulleau
}

\section{- To cite this version:}

Christian D.C. Glattli, P. Roulleau. Hanbury-Brown Twiss noise correlation with time controlled quasi-particles in ballistic quantum conductors: HIGHLIGHTS : The robust antibunching of indistinguishable leviton charge pulses is demonstrated.. Physica E: Low-dimensional Systems and Nanostructures, 2016, 76, pp.216 - 222. 10.1016/j.physe.2015.10.034 . cea-01485230

HAL Id: cea-01485230

https://hal-cea.archives-ouvertes.fr/cea-01485230

Submitted on 8 Mar 2017

HAL is a multi-disciplinary open access archive for the deposit and dissemination of scientific research documents, whether they are published or not. The documents may come from teaching and research institutions in France or abroad, or from public or private research centers.
L'archive ouverte pluridisciplinaire HAL, est destinée au dépôt et à la diffusion de documents scientifiques de niveau recherche, publiés ou non, émanant des établissements d'enseignement et de recherche français ou étrangers, des laboratoires publics ou privés. 


\title{
Hanbury-Brown Twiss noise correlation with time controlled quasi-particles in ballistic quantum conductors
}

\author{
D.C. Glattli *, P. Roulleau \\ Nanoelectronics group, SPEC, CEA, CNRS, Université Paris-Saclay, CEA Saclay 91191 Gif-sur-Yvette cedex, France
}

\section{H I G H L I G H T S}

- The robust antibunching of indistinguishable leviton charge pulses is demonstrated.

\section{A R T I C L E I N F O}

\section{Article history:}

Received 17 July 2015

Received in revised form

8 October 2015

Accepted 29 October 2015

Available online 30 October 2015

Keywords:

Electron quantum transport

Electron quantum optics

Single electron source

Quantum shot noise

\begin{abstract}
A B S T R A C T
We study the Hanbury Brown and Twiss correlation of electronic quasi-particles injected in a quantum conductor using current noise correlations and we experimentally address the effect of finite temperature. By controlling the relative time of injection of two streams of electrons it is possible to probe the fermionic antibunching, performing the electron analog of the optical Hong Ou Mandel (HOM) experiment. The electrons are injected using voltage pulses with either sine-wave or Lorentzian shape. In the latter case, we propose a set of orthogonal wavefunctions, describing periodic trains of multiply charged electron pulses, which give a simple interpretation to the HOM shot noise. The effect of temperature is then discussed and experimentally investigated. We observe a perfect electron anti-bunching for a large range of temperature, showing that, as recently predicted, thermal mixing of the states does not affect anti-bunching properties, a feature qualitatively different from dephasing. For single charge Lorentzian pulses, we provide experimental evidence of the prediction that the HOM shot noise variation versus the emission time delay is remarkably independent of the temperature.
\end{abstract}

(c) 2015 Published by Elsevier B.V.

\section{Introduction}

Markus Büttiker has made important contributions regarding the predictions of Hanbury-Brown Twiss (HBT) electron interference in quantum conductors using current noise correlations. A prerequisite to address current noise problems was the modeling of dc currents in multi-terminal conductors [1,2]. Using a scattering approach he was able to relate the current $I_{\alpha}$ injected in a contact $\alpha$ to the voltages $V_{\beta}$ applied to another contact $\beta$, i.e. $I_{\alpha}=G_{\alpha, \beta} V_{\beta}$. Büttiker's multiterminal conductance formula has given a useful frame to understand the meaning of voltage drops in quantum conductors or to implement decoherence modeling [3]. The formulation also had important impact on the practical description of edge states in the quantum Hall effect [4]. Soon after this work Markus Büttiker applied the multi-terminal approach to current fluctuations or shot noise [5]. In a long article [6],

\footnotetext{
* Corresponding author.

E-mail addresses: christian.glattli@cea.fr (D.C. Glattli), preden.roulleau@cea.fr (P. Roulleau).
}

exploiting the multi-terminal approach he made very enlightening comparisons between experimental situations encountered in quantum optics with photons and the physics of current noise cross-correlations with electrons. In this analogy a voltage biased contact plays the role of the photon source while a contact absorbing electrons the role of the photon receiver. He proposed "electron quantum optics" experimental situations, like the generic Hanbury Brown Twiss optical experiment [7] where the mixing of two beams of indistinguishable particles emitted by two different voltage sources $\alpha$ and $\beta$ gives non-trivial correlation in the statistics of joint particle detection at two separate contacts $\gamma$ and $\delta$, see also [8]. In quantum conductors, measuring the particle detection statistics is better realized by measuring current fluctuations. Ref. [6] showed that the current noise correlation $\left\langle I_{y}(t) I_{\delta}\left(t^{\prime}\right)\right\rangle$ provides direct access to the electronic HBT exchange term which is made of the product of four scattering amplitude probabilities $s_{\gamma \alpha} s_{\delta \alpha}^{*} s_{\delta \beta} s_{\gamma \beta}^{*}$. This quantity is in general not separable as a product transmission probabilities. Here $s_{i j}$ is the probability amplitude of a particle emitted from contact $i$ to reach contact $j$.

The electron quantum optics approach of [6] has stimulated a large number of experimental and theoretical works, see [9] for a 
review. The noiseless electron injection due to Fermi statistics gave rise to observation of negative shot noise correlation as expected from the partitioning of electrons between different contacts $[10,11]$. The Fermi statistics also leads to electron anti-bunching, contrasting with photon bunching. This is observed as a suppression of the cross-correlation noise in quantum conductors in a HBT like set-up where two voltage sources emit electrons in the same quantum channel [12]. In order to compare with optical Hong Ou Mandel (HOM) experiments a time control is required [13]. Continuous voltage sources do not allow us to control the emission time of particles and so to introduce a time delay between them as in the original optical HOM experiment. Markus Büttiker addressed time control in an article where he considered two sinewave voltage sources [15]. He found that the two-particle exchange term in the shot noise periodically depends on the delay between the sources. HBT and HOM experiments will be the focus of the present paper and particularly addressing on-demand quasi-particle sources.

The study of current fluctuations, i.e. time variation of the current, also pushed Markus Büttiker to be early interested in a finite frequency formulation of the multiterminal conductance formula $I_{\alpha}(\omega)=G_{\alpha, \beta}(\omega) V_{\beta}(\omega)$ [14]. By emphasizing the role of displacement currents associated to the internal potential in the conductor he provided a consistent gauge invariant formulation. He introduced the notion of emittance which allows us to take into account quantum inductance (i.e. kinetic inductance) and quantum capacitance effects. He particularly considered the situation where a quantum capacitor is weakly connected to a quantum lead via a single electronic channel of transmission $D$. While we would naively expect the RC-circuit resistance $h / D e^{2}$ from the Landauer formula, he predicted a remarkable resistance quantization even for low transmission as long as the electrons keep full quantum coherence in the capacitor. This so-called charge relaxation resistance, that we will call Büttiker resistance, is half the resistance quantum $R_{B}=h / 2 e^{2}$ (spin degeneracy is here disregarded). This has been later verified in experiments lead by one of the author, see [16].

The quantum capacitor has been the starting point towards new electron optics experiments. Beyond the ac linear capacitive response, by operating Büttiker's mesoscopic capacitor in a nonlinear regime, an ac current can be generated made of an alternate stream of emitted single electrons and single holes [17]. This is achieved by pulsing the capacitive gate voltage to periodically push and pull the last occupied energy level above and below the Fermi energy. The single particle emission time can be viewed as the time of a RC circuit. In the coherent but the non-linear regime, the associated charge relaxation resistance is no longer quantized but equals that expected in an incoherent regime $\left(\frac{1-D}{D}+\frac{1}{2}\right) h / e^{2}$, i.e. the Landauer resistance plus the access resistance of a single contact [18]. For unit transmission $D=1$, no level quantization in the capacitor, Büttiker's resistance $R_{B}=h / 2 e^{2}$ is recovered. Single electron sources provided by either a mesoscopic capacitor and by the recent voltage pulse source became one of the main focus in the contributions of Markus Büttiker and his team [19-23]. They particularly addressed HBT and HOM electron correlations, in close connection with experiments [24-29], extending very far the first electron optics shot noise predictions of Markus Büttiker.

In the present work, we would like to discuss new experimental results related to Markus Büttiker's early work where he considered HOM experiments with ac biased voltage contact [15]. We compare the sine-wave drive case to the recent Lorentzian voltage pulse case giving rise to minimal excitation states [31-35] called levitons [28]. Single and multiple charge pulses are considered both theoretically and experimentally. The paper is organized as follows. In Section 2 we discuss how to implement shot noise measurements of Hanbury Brown Twiss correlation in quantum conductors. In Section 3 we introduce basic results for the expected shot noise when the contacts are driven by ac voltage and specifically discuss the Hong Ou Mandel correlation of electron-hole pairs generated by sine-wave voltages. Section 4 discusses the case of voltage driven by periodic Lorentzian pulses giving rise to clean integer charge excitations called levitons. We propose a set of orthogonal wave-functions defined on the pulse period which allows us to give a simple interpretation of the HOM shot noise for single but also multiple electron Lorentzian pulses. Section 5 discusses the effect of temperature and presents experimental HOM measurements showing that, following the recent general prediction by Moskalets and Haack [36], this issue, the thermal mixing of the states cannot affect the HOM noise for zero emission time delay, a result contrasting with the effect of decoherence. We also show that for levitons carrying single charge the shape of HOM shot noise versus time is remarkably independent of the temperature. Section 6 is the conclusion.

\section{Measuring Hanbury Brown Twiss correlations in ballistic conductors}

In this section we discuss the conditions to realize a HOM experiment with electrons. A HOM experiment measures HBT correlations, but contrary to the original HBT measurements the thermodynamic sources randomly emitting particles are replaced by sources providing time-resolved particle emission. Examples of such sources are the optical parametric down conversion of light creating pairs of photons and the on-demand electron sources for electrons.

In the mid-1990s, following early shot noise experiments demonstrating noiseless electron flow due to Fermi statistics [37,38], several experimental groups [10-12] have addressed HBT shot noise correlations to test the electron anti-bunching predictions $[5,6,8]$. These experiments used continuous voltage sources which can be viewed as black-body sources of electrons. At zero temperature, a contact biased at a voltage $V$ above the voltage of all other contacts can be thought as emitting electrons in each quantum channel at a rate $\mathrm{eV} / \mathrm{h}$. This corresponds to a current $g e(e V / h)$ per quantum where $g=2,1$ depends on spin degeneracy. The current coming from each channel is in general partitioned by the conductor towards different output contacts. The resulting quantum partition of emitted electrons gives negative cross-correlation between currents of the output contacts. When a second contact is biased with the same voltage, the newly emitted electrons mix in the conductor with those emitted from the first contact. As electrons obey Fermi statistics, a significant reduction of the cross-correlation current fluctuations occurs due to antibunching. This effect is the fermionic counterpart of the noise doubling observed in an optical HOM experiment (or equivalently a dip in the photon coincidence detection). Although there is no doubt that anti-bunching is what was measured in experiments, the lack of time control prevents a complete parallel with Hong Ou Mandel experiments with photons. A more appropriate evidence of anti-bunching requires sending particle one by one from each source and control their arrival time in the mixing region. When the relative delay between particle $\tau$ is zero, electrons (photons) are indistinguishable and antibunching (bunching) for electrons (photons) is expected. On the opposite, for $\tau$ larger than the extension of the particle wave-packets independent partition gives no anti-bunching (no bunching) as the particle are discernable by their different arrival time at the detectors. The comparison between these two limiting case provides a clean way to quantify bunching effects.

Not related to the present discussion, another advantage of 
controlling the particle emission time is the measurement of the overlap of the particle wave-functions by varying $\tau$, see Sections 4 and 5 . This provides a way to measure the wave-packet shape and its width (actually the initial motivation of Hong, Ou and Mandel) [28,27]. This provides also information on decoherence mechanisms in the case of strong decoherence effects as in [27].

Another drawback of using continuous voltage sources is related to gauge invariance which trivially prevents to observe antibunching with a 2-terminal conductor (where the contact are both used to emit and detect the currents) as applying the same voltage on both contacts leads to no current and thus no noise. This is no longer the case if instead of using continuous sources, the voltages are time dependent. At finite frequency a conductor with two ohmic contacts can no longer be viewed as a two-terminal conductor. This is clear from Büttiker's multiterminal ac conductance relations $I_{\alpha}(\omega)=G_{\alpha, \beta}(\omega) V_{\beta}(\omega)$ which include displacement currents to restore gauge invariance [14]. At the lowest order in frequency taking displacement currents into account requires a third electrode with electrochemical capacitance $C_{\mu}=C_{q} C /\left(C_{q}+C\right)$. Here $C$ is the geometrical capacitance and $C_{q}$ the quantum capacitance related to the electron density of state. Indeed, consider the simplest situation of a ballistic conductor with two ohmic contacts made of a single channel with a punctual scatterer in its middle. Rewriting left and right currents $I_{L(R)}$ and voltages $V_{L(R)}$ in time-domain , one gets $I_{L}(t)=\frac{g e^{2}}{h}\left(V_{L}(t)-(1-D) V_{L}\left(t-\tau_{L L}\right)-D V_{R}\left(t-\tau_{L R}\right)\right)$ and similarly for $I_{R}(t)$, where $\tau_{L L}$ and $\tau_{L R}$ are the propagation time of backscattered and transmitted electrons and $D$ is the transmission of the scatterer. It is thus obvious that applying the same voltage $V(t)=V_{L}(t)=V_{R}(t) \quad$ results in a finite injected current $\frac{g e^{2}}{h}\left(V(t)-(1-D) V\left(t-\tau_{L L}\right)-D\left(t-\tau_{R L}\right)\right)$ and a displacement current $d Q / d t=I_{L}(t)+I_{R}(t)$. Applying the same voltage on opposite ohmic contacts is not like doing nothing. For example, there is a finite dissipated power which is given by the time average of $\frac{g e^{2}}{h}\left(V(t)-V\left(t-\tau_{p}\right)\right)^{2}$ (a single propagation time $\tau_{p}$ is taken for simplicity). Propagation times like $\tau_{R L}$ have been measured in a two-terminal quantum conductor in the quantum Hall regime in Ref. [30].

Fig. 1 shows two generic equivalent situations for HOM measurements, one using chiral propagation as it can be realized using edge states of the QHE, the other one in a non-chiral two-terminal configuration. Both give identical information.

\section{Electron-hole pair Hong Ou Mandel correlations using sine- wave voltage sources}

We will first recall the case of two sine-wave voltages sources as considered by Büttiker [15]. A voltage $V(t)_{1(2)}=V_{a c} \cos \left(2 \pi \nu t+\phi_{1(2)}\right)$ is applied on two contacts with relative phase shift $\Delta \phi=\phi_{1}-\phi_{2}=2 \pi \nu \tau$ and frequency $\nu$. Here the contact labels correspond to those shown in Fig. 1(a). Denoting the HBT scattering phase $\chi=\arg \left(S_{13}^{\dagger} S_{32} S_{24}^{\dagger} S_{41}\right)$, the low frequency cross correlation of the current at contacts 3 and 4 found in [15]:

$S_{I_{3} I_{4}}(\Delta \phi)=-e^{2} \nu P_{1}\left|S_{13}^{\dagger} S_{41} e^{-i \Delta \Phi}+S_{23}^{\dagger} S_{42}\right|^{2}$

In the case of a punctual scatterer like in Fig. 1, $\chi=\pi+2 \pi \nu\left(\tau_{32}-\tau_{31}+\tau_{41}-\tau_{32}\right)$, where $\tau_{\beta \alpha}$ is the time to propagate from contact $\alpha$ to contact $\beta$. The $\pi$ phase shift comes from the scatterer described by a unitary scattering matrix. For $\Delta \phi=\chi+\pi$, mod. $2 \pi$, the shot noise vanishes. This corresponds to zero phase shift between the voltage sources $(\tau=0)$ : the created excitations are indistinguishable and perfect anti-bunching occurs as expected for fermionic excitations. For $\tau=T / 2(T=1 / \nu$ the period $)$ the noise is maximal as the excitations being not created at the
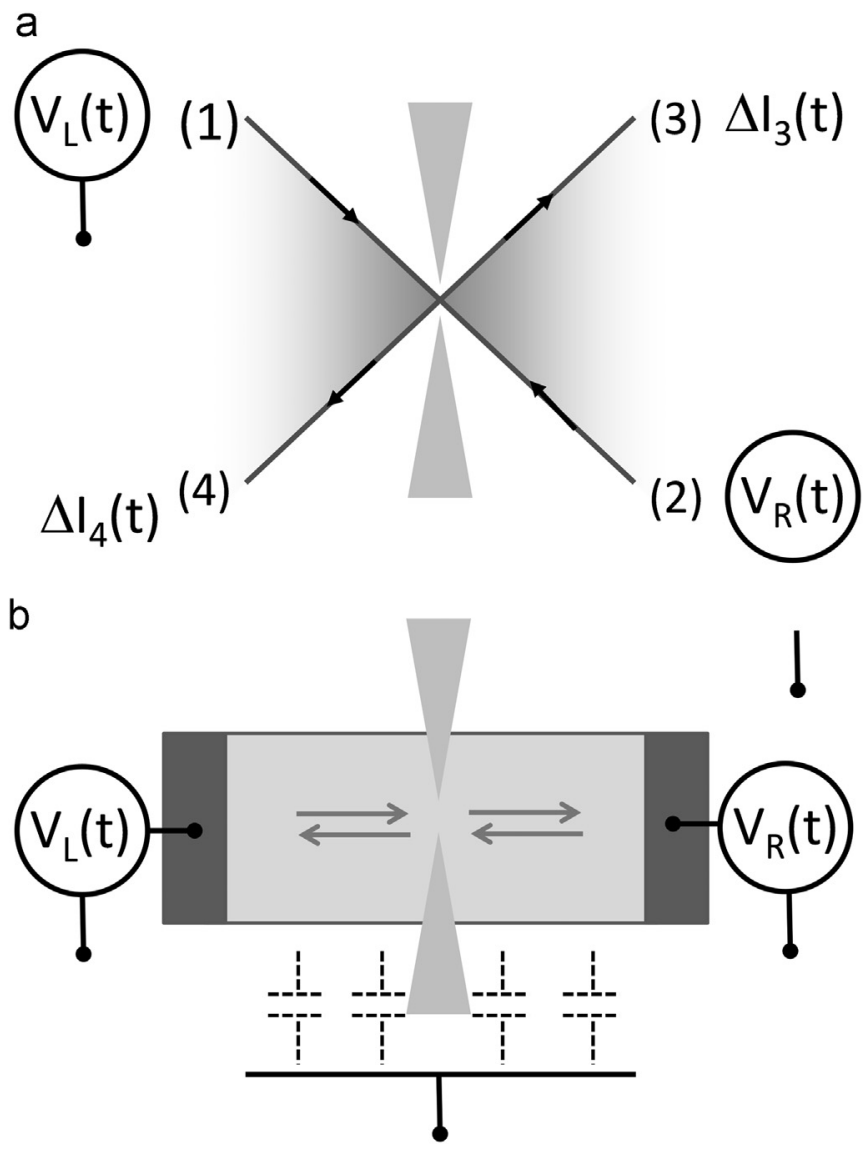

Fig. 1. Two generic equivalent quantum conductor set-up for probing Hong Ou Mandel correlations. (a) Chiral propagation using, for example, the edge states of the quantum Hall effect. Voltage are applied at the upper left and lower right contact to inject electronic excitations. The cross correlation of the current fluctuations are made at lower left and upper right contacts to probe the correlations of outgoing particles. (b) Non-chiral sample. Here, the injected quasi-particle is reflected back to the emitting contact. At finite frequency, the conductor is no longer a two-terminal conductor as displacement current, necessary to ensure current conservation connects the sample to nearby capacitor. In both (a) and (b) cases, the incoming particles can be partitioned in the output channel using a punctual scatterer, in practice a quantum point contact allows us to control the transmission $D$ and so the partitioning factor $D(1-D)$ leads to shot noise.

same time become distinguishable. These two limiting cases correspond to those of a HOM experiment. Ref. [15] considered a small voltage amplitude $V_{a c} \ll h_{\nu} / e$. The sine-wave excitation creates electron-hole pairs of energy $h_{\nu}$ with a small probability $P_{1}=J_{1}\left(e V / 2 h_{\nu}\right)^{2} \approx(e V / 2 h \nu)^{2} \ll 1$.

In the following we will consider voltage pulse sources injecting an integer number of electron ne per period $T=1 / \nu$ in a single electron channel (spin is disregarded in this section). Now the voltage applied on the injecting contacts is of the form $V(t)=V_{d c}+V_{a c} \cos (2 \pi \nu t+\phi)$. The condition to inject charge ne requires $e V_{d c}=n h \nu$, see for example discussion in [39,40]. The condition to have the appropriate pulse amplitude requires a relation linking $V_{a c}$ to $V_{d c}$ as shown for particular examples in the following. For sine-wave pulses it is $V_{a c}=V_{d c}$.

Considering first a single biased contact, say 1 , the current $I_{0}(t)=n e \nu(1+\cos (2 \pi \nu t+\phi)$ is injected and partitioned with transmission $D$ and reflection $1-D$ towards contacts 3 and 4 . Adapting the Floquet scattering theory of Ref. [41], the low frequency shot noise observed at one output contact is given by [39]:

$S_{I}=2 \frac{e^{2}}{h} D(1-D)\left[\sum_{l=-\infty}^{+\infty}\left|p_{l}\right|^{2}\left|l h \nu+e V_{d c}\right|\right]$ 
where for sine-wave pulses $p_{l}=J_{l}\left(e V_{a c} / h_{\nu}\right)$ and $J_{l}$ is an integer Bessel function. Most of the physics is contained in the probability amplitude $p_{l}$ to find an electron emitted at any energy $\varepsilon$ below the Fermi energy of the contact to be promoted at an energy $\varepsilon+l_{\nu}$ when experiencing the ac voltage. The latter periodically modulates the phase of the electrons $\varphi(t)=2 \pi e \int^{t} V_{a c}\left(t^{\prime}\right) d t^{\prime} / h$ and $p_{l}=1 / T \int_{0}^{T} \exp (i l \nu t) \exp (-i \varphi(t)) d t$.

A more general expression, including finite temperature, can be obtained by rewriting Eq. (2) as:

$S_{I}=\sum_{l=-\infty}^{\infty}\left|p_{l}\right|^{2} S_{I}^{d c}\left(l h \nu+e V_{d c}\right)$

where $S_{I}^{d c}$ is the shot noise that would be observed if only a dc bias voltage $V$ were applied on the left contact. This dc shot noise expression is well known and given by:

$S_{I}^{d c}(V)=2 e V\left(g e^{2} / h\right) D(1-D) \operatorname{coth}\left(e V / 2 k_{B} T_{e}\right)+4 k_{B} T_{e} g D^{2} e^{2} / h$

Lets us now consider the HBT correlations in a Hong Ou Mandel like set-up where the same excitation is applied on both contacts but with a time delay $\tau$ corresponding to phase shifts $\phi_{1}=0$ and $\phi_{2}=2 \pi \nu \tau$. The probability amplitudes for electron or hole creation from the left and right injecting contacts are $p_{l}$ and $p_{l}^{\prime}=p_{l} e^{i 2 \pi \nu \tau}$ respectively. The noise is given by Eq. (3) with the $p_{l}$ replaced by $\Pi_{l}=\sum_{k} p_{k-l} p_{k}^{\prime *}[39]$. Explicitly:

$\Pi_{l}(\tau)=\sum_{k} p_{k-l} p_{k}^{*} e^{-i k 2 \pi \nu \tau}$

and

$S_{I}^{H O M}=\sum_{l=-\infty}^{\infty}\left|\Pi_{l}\right|^{2} S_{I}^{d c}(l h \nu)$

Note that $V_{d c}$ has dropped in the above expression. From Eq. (5), using the addition theorem of Bessel functions one finds $\Pi_{l}=J_{l}\left(2 e V_{a c} \sin (2 \pi \nu \tau / 2) / h_{\nu}\right)$. It is easy to check that one recovers the result of Ref. [15] discussed above for sine-wave excitations where $p_{l}=J_{l}\left(e V / 2 h_{\nu}\right)$. For weak excitation voltage, only the zero and first order Bessel functions are significant and to the lowest order the only term $\left|\Pi_{l}\right|^{2}$ contributing to $(5)$ is $\left|\Pi_{l}\right|^{2} \approx(e V / 2 h \nu)^{2}\left|e^{i 2 \pi \nu \tau}-1\right|^{2}$ leading to

$S_{I}(\tau) \simeq(e V / h \nu)^{2} \sin (2 \pi \nu \tau)^{2} S_{I}^{d c}(h \nu / e)$

\section{Multiple Hong Ou Mandel correlations using Lorentzian voltage pulses}

The sinewave voltage sources considered above provide a coherent source of electron-hole pairs with energy multiple of $h \nu$. The combination of a dc voltage $V_{d c}=n h \nu / e$ and an ac voltage $V_{a c}=V_{d c}$ can be viewed as the periodic introduction of ne charge per pulses in the conductor. However it does not produce clean charge pulses, i.e. the number of excitations is larger than the number of charges. Indeed for any voltage pulse shape it can be generally shown that Eq. (3) can be rewritten as $S_{I}=2 \nu e^{2}\left(N_{e}+N_{h}\right)$ where $N_{e / h}$ is the number of electrons or holes injected per period by the voltage pulse, while the average current is e $\nu\left(N_{e}-N_{h}\right)=n e \nu$. A clean excitation would require $N_{h}=0$. For sine-wave pulses one finds $N_{h}$ equal to $\simeq 0.057$ and $\simeq 0.08$ for $n=1$ and 2 respectively. For square wave pulses a larger amount is found ( $\simeq 0.21$ and $\simeq 0.34$ respectively). As theoretically proposed in [31-34] and recently experimentally observed [28,29], the only way to generate clean integer charge pulses, i.e. $N_{h}=0$, is to apply Lorentzian pulses. The resulting clean time-resolved coherent excitations are minimal excitation states called levitons. Another possibility is to use the mesoscopic capacitor source [17] in the adiabatic regime where, by slowly increasing the capacitive gate voltage, the last occupied energy level adiabatically crosses the Fermi level [42]. In practice this is very demanding experimentally regarding available time and energy scales of typical quantum dots and the emission is limited to single charge. Lorentzian voltage pulses are much easier to operate.

In the following we discuss the HBT correlation when injecting clean integer charge pulses (levitons). We will consider zero temperature. The HOM shot noise can then be simply interpreted as given by a measure of the overlap of the wavefunctions of levitons emitted by each contact with the relative delay $\tau$. For periodic injection we introduce a new wavefunction basis which provides simple interpretation and an efficient way to compute the HOM shot noise for any number of electrons.

Let us first consider a single leviton obtained by applying the voltage $V(t)=\left(h_{\nu} / e \pi\right) 1 /\left(1+t^{2} / W^{2}\right)$ on one contact. The electron phase modulation is given by $\exp (-i \phi(t))=(1+i W) /(1-i W)$. The envelope wavefunction (i.e. disregarding the factor $\left.e^{i E_{F}\left(t-x / v_{F}\right) / \hbar}\right)$ of the leviton created above the Fermi sea is [33]:

$\psi\left(t-x / v_{F}\right)=\frac{1}{\sqrt{\pi v_{F} W}} \frac{1}{1+\left(t-x / v_{F}\right)^{2} / W^{2}}$

where $v_{F}$ is the Fermi velocity and $2 W$ the full width at mid-height of the Lorentzian voltage pulse. If two similar pulses are injected from left and right contact with respectively the time delay $\mp \tau / 2$, and the experiment is repeated at a low frequency $\nu \ll W^{-1}$ such that pulse overlap can be disregarded, the HOM shot noise is [39]:

$S_{I}^{\text {HOM }}=4 \nu e^{2} D(1-D)(1-g(\tau))$

where $g(\tau)=|<\psi(t+\tau / 2)| \psi(t+\tau / 2)>\left.\right|^{2}$

For $\tau \gg W$ (and $\tau$ assumed smaller that the period $T$ ) Eq. (9) expresses the shot noise of two independent levitons partitioned by the scatterer of transmission $D$, while for $\tau=0$, full overlap of the wave functions, electron anti-bunching due to Fermi statistics gives zero noise.

Can relation (9) be generalized at zero temperature to the case of periodic pulses for which the overlap cannot be neglected? Clearly the wavefunctions given by Eq. (8) are no longer appropriate as they extend over more than a period. It will be better to find a basis of wavefunctions defined over the period $T=\nu^{-1}$.

For periodic voltage pulses injecting levitons of charge ne:

$V(t)=\frac{V_{a c}}{\pi} \sum_{k=-\infty}^{+\infty} \frac{1}{1+(t-k T)^{2} / W^{2}}$

where $e V_{a c}=n h \nu$. The total phase $\Phi(t)$ acquired by electrons emitted by the driven contact is given by:

$\exp (-i \Phi(t))=\left(\frac{\sin (\pi(u+i \eta))}{\sin (\pi(u-i \eta))}\right)^{n}$

where reduced time units $u=t / T$ and $\eta=W / T$ are used. For single charge leviton $n=1$, the $p_{l}$ and $\Pi_{l}$ have been calculated, see for example [39]. Now let us consider the HOM noise expected when single charge levitons are sent from left and right contacts with delay $\pm \tau / 2$ respectively. It can be obtained from Eq. (6) [39]:

$S_{I}^{H O M}(\tau)=4 e^{2} \nu D(1-D) \frac{2 \beta^{2}\left(1-\cos (2 \pi \nu \tau)^{2}\right.}{1-2 \beta^{2} \cos (2 \pi \nu \tau)+\beta^{4}}$

where $\beta=\exp (-2 \pi W / T)$ characterizes the width of the Lorentzian.

In order to express the noise in the form of Eq. (9), we conjecture that an appropriate wavefunction defining a single leviton over the period $T$ and generalizing Eq. (8) is given by: 
$\psi(u)=\frac{1}{\sin (\pi(u-i \eta))}$

From it one can calculate $g(\tau)$ as:

$$
\begin{aligned}
g(\tau) & =\left|\int_{-1 / 2}^{1 / 2} \psi(u+\tau / 2 T) * \psi(u+\tau / 2 T) d u\right|^{2} \\
& =\frac{\left(1-\beta^{2}\right)^{2}}{\left(1-2 \beta^{2} \cos (2 \pi \nu \tau)+\beta^{4}\right.}
\end{aligned}
$$

We can check that this form of $g(\tau)$ agrees with Eq. (12) with $S_{I}^{H O M}(\tau)=4 e^{2} \nu D(1-D)(1-g(\tau))$.

Encouraged by this result we can define a set of orthogonal wavefunctions $\psi_{k}, k=0,1,2, \ldots$, defined on the period $T$ to build periodic trains of levitons carrying $n$ charges:

$\psi_{k}(u)=\frac{(\sin (\pi(u+i \eta)))^{k-1}}{(\sin (\pi(u-i \eta)))^{k}}$

For example, the two-leviton wavefunction built from a Slater determinant involving $\psi_{0}(u)$ and $\psi_{1}(u)$ allows us to directly calculate the HOM shot noise when periodic trains of 2e-levitons are injected from left and right contacts with relative time delay $\pm \tau / 2$. In that case one expects:

$S_{I}^{H O M}(\tau)=4 e^{2} \nu D(1-D)\left(2-g^{(1)}(\tau)-g^{(2)}(\tau)\right)$

where $g^{(k)}(\tau)=\left|\left\langle\psi_{k}(\tau / 2) \mid \psi_{k}(-\tau / 2)\right\rangle\right|^{2}$

Using this approach, we obtain the expression for the 2e-leviton HOM shot noise:

$S_{I}^{H O M}(\tau)=4 e^{2} \nu D(1-D) \lambda(\tau) \frac{\left(2+\lambda(\tau)+\lambda(\tau)^{2}\right)}{(1+\lambda)^{3}}$

where $\lambda(\tau)=\left(2 \beta \sin (\pi \nu \tau) /\left(1-\beta^{2}\right)\right)^{2}$. We have checked that this expression coincides with a direct, but lengthy, calculation based on photon-assisted processes using Eq. (5) where the $\Pi_{l}$ are calculated from the $p_{l}$ of 2e-levitons (explicitly $p_{l}=\beta^{l}\left(1-\beta^{2}\right)\left(1-3 \beta^{2}+l\left(\left(1-\beta^{2}\right)\right)\right)$.

It is interesting to consider the limit of well separated doubly charged levitons. In this limit $W / T \ll 1$, no overlap of pulses, $\psi_{k}=(t+i W)^{k-1} /(t-i W)^{k}$ and for useful time delay $\tau \ll T, \lambda \simeq \tau / 2 W$.

To conclude this section, we have shown that at zero temperature HBT correlations of trains of periodic leviton pulses for arbitrary charge can be described by simple wavefunctions defined over the period which allows us to calculate multiple HOM shot noise correlations. The exploration of the possibilities to make multiple electron pulse interferences is an exciting field which will deserve further theoretical and experimental investigations.

In the next section we show measurements up to $n=2$ and discuss the effect of the temperature.

\section{Temperature dependence of Hong Ou Mandel shot noise with single charge pulses}

The interpretation of the shot noise in terms of single particles created above the Fermi sea could let think that the clean electron created in a single charge pulse is independent from the Fermi sea. In reality, the single particle is created from a collective excitation of the whole Fermi sea excited by the voltage pulse. It is only at zero temperature that the two pictures coincide to give similar expressions of the shot noise. At finite temperature it is mandatory to use Eq. (6).

The vanishing of the noise at $\tau=0$ signaling perfect antibunching is remarkably robust. Indeed, $\Pi_{k}(\tau=0)=\delta_{k, 0}$ which leads to $S_{I}^{H O M}(\tau=0)=0$. This contrasts with the effect of decoherence which affects the HOM dip and leads to finite shot noise at zero time delay [27]. This qualitative distinction is discussed in a contribution by Moskalets and Haack, same special issue, [36]. They make a general comparison between decoherence and thermal effects. They show that the mixed state nature of the single charge pulses at finite temperature preserves coherence and that perfect anti-bunching remains visible in the HOM shot noise. We can draw a parallel between HOM shot noise and MachZehnder interferometry. For nearly equal Mach-Zehnder interferometer arms (zero length difference) the transmission is only weakly affected by thermal excitations but can be affected by decoherence (and so indirectly by temperature through the temperature dependence of the coherence length). A complete study of the temperature dependence of the visibility of a Mach-Zehnder interferometer transmitting voltage pulses (sine-waves and Lorentzian) has been recently discussed in Ref. [43].

In the following we report HOM shot noise measurements done at various electronic temperature $T_{e}$ for single charge sinewave pulses, and single and double charge levitons. The experimental set-up is the same as the one used in Ref. [28,29], with voltage pulses applied on ohmic contact of a $2 \mathrm{D}$ electron gas made in GaAs/GaAlAs heterojunction. A quantum point contact is used to realize the punctual scatterer partitioning the charge pulses. The transmissions used are in the range $D \simeq 0.3-0.35$. The cross-correlation of the current fluctuations is measured with sub-femtoampere resolution using cryogenic amplifiers. The base electronic temperature is $\approx 30 \mathrm{mK}$. Experimental details regarding the setup can be found in Refs. $[28,29]$ and the associated supplementary information.

Fig. 2(a), shows HOM shot noise measurements for single charge levitons for three temperatures $T_{e}=40 \mathrm{mK}, 90 \mathrm{mK}$ and $138 \mathrm{mK}$ (dots). The repetition rate is $\nu=4.8 \mathrm{GHz}$ and $W=37.5 \mathrm{ps}$ ( $W / T=0.18$ ). For $\tau=0$ one observes that for all three temperatures the shot noise vanishes showing that coherence is preserved and that finite temperature does not affect perfect anti-bunching. For $\tau=T / 2$, maximum separation between pulses the noise is the largest as the wave-function overlap is the smallest. The theoretical values of shot noise $S_{I}^{H O M}\left(\tau, T_{e}\right)$ obtained from Eq. (3) using the appropriate $\Pi_{l}$ are shown as solid line curves and perfectly match the data with no adjustable parameters.

For single charge levitons another remarkable effect is that thermal effects do not prevent to get exact information on the temporal shape of the wave function lines. As predicted in [39] there is a full decoupling between the temperature and the $\tau$ dependence of the HOM shot noise:

$S_{I}^{H O M}\left(\tau, T_{e}\right)=F\left(k_{B} T_{e} / h_{\nu}\right) S_{I}^{H O M}(\tau, 0)$

The reason is the multiplicative property of the $p_{l}$ for single charge levitons leading to $\Pi_{l}(\tau)=\beta^{l} \Pi_{1}(\tau)$ for $l \neq 0$ such that the $\tau$ dependence factorizes. This remarkable feature is well experimentally observed and shown in Fig. 2(b) where we have taken the HOM curve at $90 \mathrm{mK}$ temperature and plotted its ratio to the zero temperature expression, i.e. $S_{I}^{H O M}\left(\tau, T_{e}=90 \mathrm{mK}\right) / S_{I}^{H O M}(\tau, 0)$. The result is a flat curve demonstrating that the two original curves are homothetic (note that for $\tau$ close to 0 or $T$ as both curves go to zero the error bars diverge).

We now consider sine-wave pulses carrying integer charge. Fig. 3(a) shows HOM shot noise measurements for two temperatures $T_{e}=89 \mathrm{mK}$ and $138 \mathrm{mK}$. A first remarkable feature is again the vanishing noise for $\tau=0$ and $T$. This shows that even though sinewave pulses do not produce minimal excitation states, as all excitations being fermionic, they perfectly antibunch and this effect is not affected by temperature (assuming coherence preserved). But contrary to the case of single charge levitons there is no longer decoupling between the temperature and the $\tau$ dependence of the HOM shot noise. Indeed, the $\Pi_{l}$, being now equal to 
a

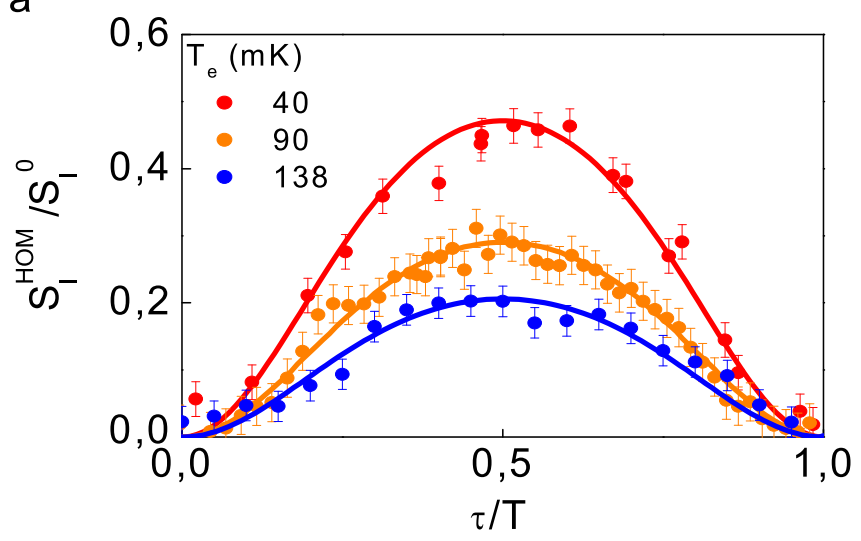

b

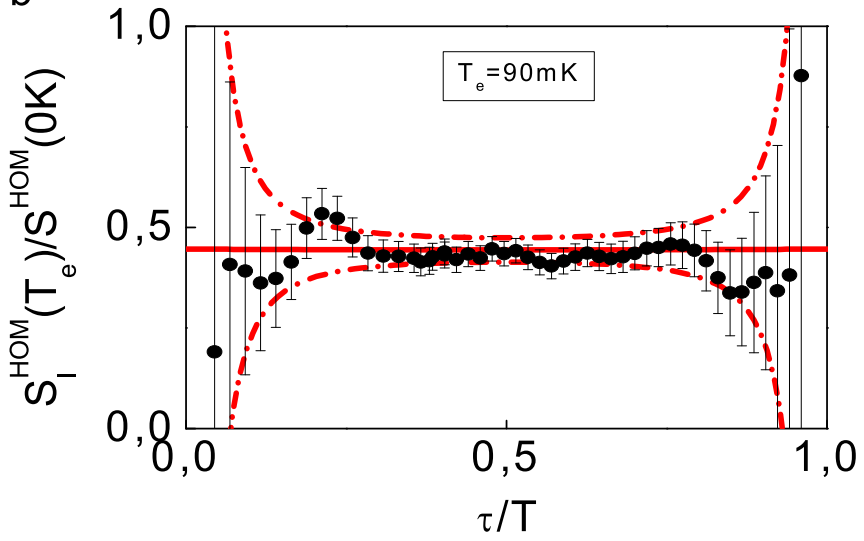

Fig. 2. (a) Dots show measured HOM noise versus time delay for a Leviton at three different temperatures $(40,90$ and $138 \mathrm{mK}$ ) and transmissions $D=0.34,0.31$ and 0.31 respectively. The theory is represented in solid lines (including joule heating effect). (b) The black dots show the ratio of the measured HOM shot noise at $T_{e}=90 \mathrm{mK}$ to the zero temperature HOM shot noise versus $\tau$, i.e. $S_{I}^{H O M}\left(\tau, T_{e}\right) / S_{I}^{H O M}(\tau, 0 \mathrm{~K})$. As expected, we do not observe any dependence of this ratio with $\tau$. Thermal fluctuations do not prevent from having a precise measurement of the time shape of the wavefunction, it only affects the amplitude of the signal. Red dashed line shows the theoretical error bar (error bars diverge for $\tau=0$ or $T$ since $S_{I}^{H O M}(\tau, 0 \mathrm{~K})=0$ ). (For interpretation of the references to color in this figure caption, the reader is referred to the web version of this paper.)

$J_{l}(2 \sin (\pi \nu \tau)$, do not show factorization. Fig. 3(b) shows the ratio of the data curve taken at $T_{e}=89 \mathrm{mk}$ to the theoretical curve at $T_{e}=0$. The resulting curve is no longer flat showing that the two curves are not homothetic. The solid line curves in Fig. 3(a) are the computation at finite temperature. There is no adjustable parameters and the agreement is excellent.

Finally we consider multiple HOM shot noise correlation with doubly charged levitons in Fig. 4(a). The width and repetition frequency are the same for the data shown above for single charge levitons. The three curves correspond to temperature $T_{e}=40,90$ and $138 \mathrm{mK}$. Again excellent and thermally insensitive antibunching is observed for $\tau=0$ and $T$ as the noise vanishes. The agreement between data and theory, solid curves, is again excellent. The amplitude probabilities for double charge levitons are given by $p_{l}=\beta^{l}\left(1-\beta^{2}\right)\left(1-3 \beta^{2}+\left(1-\beta^{2}\right)\right)$ and no longer show multiplicative property, except in the limit $\beta \rightarrow 1$ (sharp pulses with no overlap) and the $\Pi_{l}$ do not show the factorization property of single charge levitons. Thus HOM curves versus $\tau$ at finite temperature are not proportional the zero temperature curve as shown in Fig. 4(b) where the ratio $S_{I}^{H O M}\left(\tau, T_{e}=90 \mathrm{mK}\right) / S_{I}^{H O M}(\tau, 0)$ is plotted. a
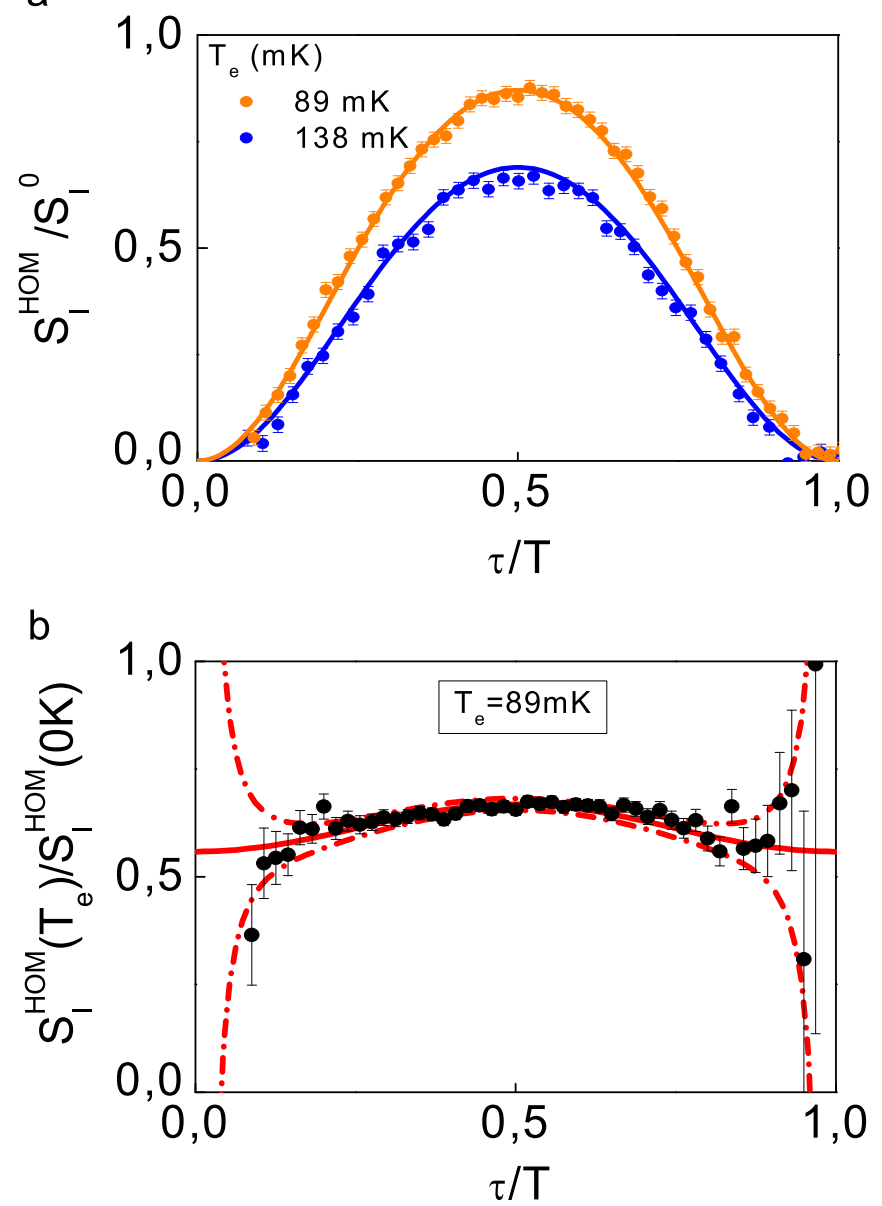

Fig. 3. (a) Dots show the measured HOM noise versus time delay for sine pulse carrying a single charge for two different temperatures $89 \mathrm{mK}$ and $138 \mathrm{mK}$ and for transmission respectively equal to $D=0.35$ and 0.31 . The theory is represented in solid lines. (b) Measured (black dots) $S_{I}^{H O M}\left(\tau, T_{e}\right) / S_{I}^{H O M}(\tau, 0 \mathrm{~K})$ as a function of $\tau$ for $T_{e}=88 \mathrm{mK}$ is compared with the theory (red solid line). Contrary to the Leviton, we observe a dependence of this ratio with $\tau$. Red dashed line shows the theoretical error bar (it diverges since $S_{I}^{H O M}\left(\tau, T_{e}\right) / S_{I}^{H O M}(\tau, 0 K)=0$ for $t=0$ or $T$ ). (For interpretation of the references to color in this figure caption, the reader is referred to the web version of this paper.)

\section{Conclusion}

We have considered the Hanbury Brown Twiss correlation of electrons in a quantum conductor in the situation where the time control of electrons or of electron-hole pairs allows us to perform the electronic analog of Hong Ou Mandel measurements. We have only considered time resolved excitations generated by applying time dependent voltage on the contacts of the conductor and specifically sinewave and Lorentzian voltage pulses. For the case of Lorentzian voltage pulses periodically injecting single or multiple electrons in the form of the minimal state called leviton, we have proposed a set of orthonormal wavefunctions defined over the period which allows us to generalize the interpretation of HOM shot noise in terms of a measure of the overlap of single particle wavefunctions. We have then discussed the thermal effect and experimentally shown that electron anti-bunching due to Fermi statistics is robust to thermal effects. We have also shown that the shape of the HOM shot noise of single charge levitons versus time delay is not affected by temperature except its amplitude. 
a

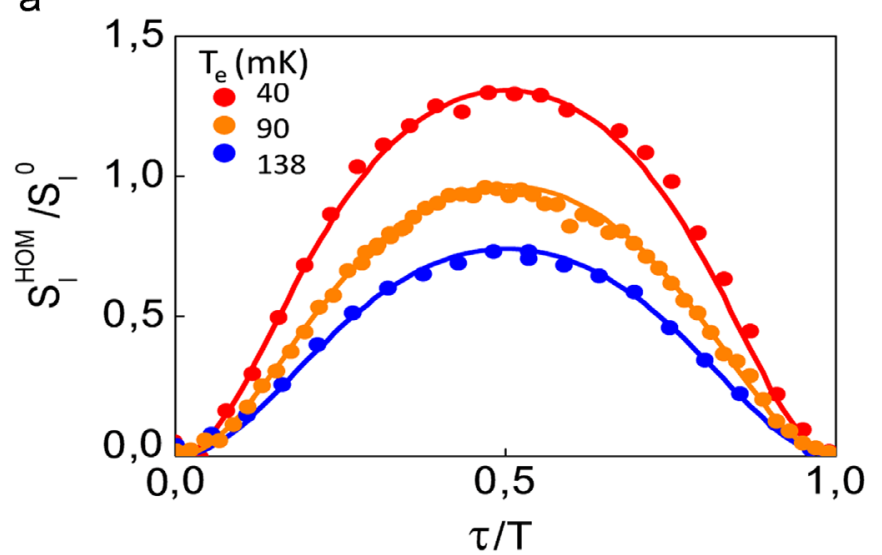

b

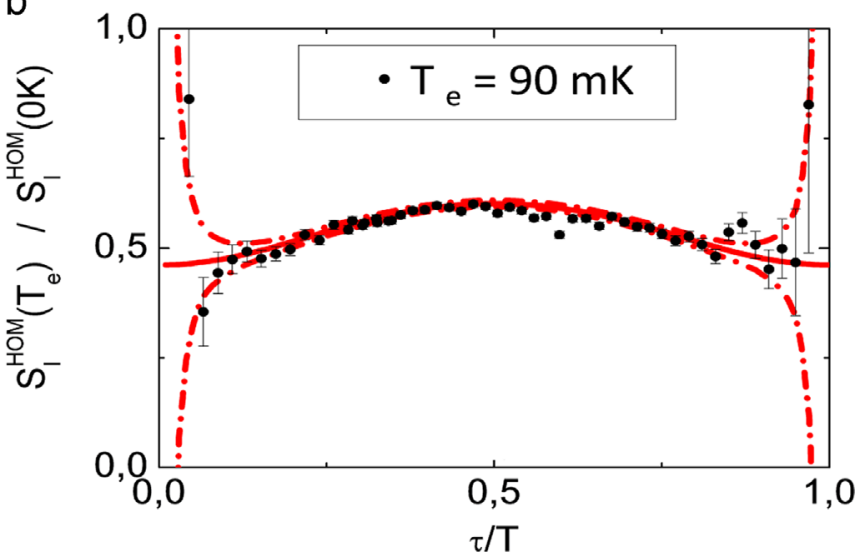

Fig. 4. (a) Dots show measured HOM noise versus time delay for Lorentzian pulse carrying two charges for three different temperatures $40 \mathrm{mK}, 87 \mathrm{mK}$ and $138 \mathrm{mK}$ and for transmission respectively equal to $D=0.34,0.31$ and 0.31 . The theory is represented in solid lines (including joule heating effect). (b) Measured (black dots) $S_{I}^{H O M}\left(\tau, T_{e}\right) / S_{I}^{H O M}(\tau, 0 K)$ as a function of $\tau$ is compared with the theory (red solid line). As expected,we observe a dependence of this ratio with $\tau$. Red dashed line shows the theoretical error bar. (For interpretation of the references to color in this figure caption, the reader is referred to the web version of this paper.)

\section{Acknowledgments}

Support from the ERC Advanced Grant 228273 MeQuaNo is acknowledged. This work has been greatly inspired by Markus Büttiker's work and his numerous collaborators. We thank M. Moskalets and G. Haack for sending their preprint.

\section{References}

[1] M. Büttiker, Phys. Rev. Lett. 57 (1986) 1761.

[2] M. Büttiker, Phys. Rev. B 33 (1986) 3020.

[3] M. Büttiker, IBM J. Res. Dev. 32 (1988) 63.

[4] M. Büttiker, Phys. Rev. B 38 (1988) 9375.

[5] M. Büttiker, Phys. Rev. Lett. 65 (1990) 2901.

[6] M. Büttiker, Phys. Rev. B 46 (1992) 12485.

[7] R. Hanbury Brown, R.Q. Twiss, Nature 177 (1956) 27.

[8] Th. Martin, R. Landauer, Phys. Rev. B 45 (1992) 1742.

[9] Y.M. Blanter, M. Büttiker, Phys. Rep. 3361 (2000).

[10] M. Henny, et al., Science 284 (1999) 296.

[11] W.D. Oliver, J. Kim, R.C. Liu, Y. Yamamoto, Science 284 (1999) 299.

12] R.C. Liu, B. Odom, Y. Yamamoto, S. Tarucha, Nature 391 (1998) 263.

[13] C.K. Hong, Z.Y. Ou, L. Mandel, Phys. Rev. Lett. 59 (1987) 2044.

[14] M. Büttiker, A. Prêtre, H. Thomas, Phys. Rev. Lett. 70 (1990) 4114.

[15] V.S. Rychkov, M.L. Polianski, M. Büttiker, Phys. Rev. B 72 (2005) 155326.

[16] J. Gabelli, G. Fève, J.-M. Berroir, B. Plaçais, A. Cavanna, B. Etienne, Y. Jin, D. C. Glattli, Science 313 (2006) 499-502.

[17] G. Fève, A. Mahé, J.-M. Berroir, T. Kontos, B. Plaçais, D.C. Glattli, A. Cavanna, B. Etienne, Y. Jin, Science 316 (2007) 1169-1172.

[18] S.E. Nigg, M. Büttiker, Phys. Rev. B 77 (2008) 085312.

[19] M. Moskalets, P. Samuelsson, M. Büttiker, Phys. Rev. Lett. 100 (2008) 086601

[20] J. Splettstoesser, M. Moskalets, M. Büttiker, Phys. Rev. Lett. 103 (2009) 076804

[21] M. Albert, C. Flindt, M. Büttiker, Phys. Rev. B 82 (2010) 041407 (R).

[22] S. Olkhovskaya, J. Splettstoesser, M. Moskalets, M. Büttiker, Phys. Rev. Lett. 101 (2008) 166802.

[23] G. Haack, M. Moskalets, J. Splettstoesser, M. Büttiker, Phys. Rev. B. 82 (2011) 081303 (R)

[24] A. Mahé, F.D. Parmentier, E. Bocquillon, J.-M. Berroir, D.C. Glattli, T. Kontos, B. Plaçais, G. Fève, A. Cavanna, Y. Jin, Phys. Rev. B. 84 (2010) 201309 (R).

[25] F.D. Parmentier, E. Bocquillon, J.-M. Berroir, D.C. Glattli, B. Plaçais, G. Fève, M. Albert, C. Flindt, M. Büttiker, Phys. Rev. B 85 (2012) 165438.

[26] E. Bocquillon, F.D. Parmentier, C. Grenier, J.-M. Berroir, P. Degiovanni, D. C. Glattli, B. Plaçais, A. Cavanna, Y. Jin, G. Fève, Phys. Rev. Lett. 108 (2012) 196803.

[27] E. Bocquillon, V. Freulon, J.-M. Berroir, P. Degiovanni, B. Plaçais, A. Cavanna, Y. Jin, G. Feve, Science 339 (2013) 1054.

[28] J. Dubois, T. Jullien, F. Portier, P. Roche, A. Cavanna, Y. Jin, W. Wegscheider, P. Roulleau, D.C. Glattli, Nature 502 (2013) 659-663.

[29] T. Jullien, P. Roulleau, B. Roche, A. Cavanna, Y. Jin, D.C. Glattli, Nature 514 (2014) 603-607.

[30] J. Gabelli, et al., Phys. Rev. Lett. 98166806 (2007).

[31] L.S. Levitov, H. Lee, G. Lesovik, J. Math. Phys. 374845 (1996); D.A. Ivanov, H.W. Lee, L.S. Levitov, Phys. Rev. B 56 (1997) 6839.

[32] A.V. Lebedev, G.V. Lesovik, G. Blatter, Phys. Rev. B 72 (2005) 245314

[33] J. Keeling, I. Klich, L.S. Levitov, Phys. Rev. Lett. 97 (2006) 116403.

[34] L.S. Levitov, G.B. Lesovik, JETP Lett. 58 (1993) 230.

[35] F. Hassler, M.V. Suslov, G.M. Graf, M.V. Lebedev, G.B. Lesovik, G. Blatter, Phys. Rev. B 78 (2008) 165330.

[36] M. Moskalets, G. Haack, arXiv:1506.09028v1, this issue.

[37] M. Reznikov, M. Heiblum, H. Shtrikman, D. Mahalu, Phys. Rev. Lett. 75 (1995) 3340.

[38] A. Kumar, L. Saminadayar, D.C. Glattli, Y. Jin, B. Etienne, Phys. Rev. Lett. 76 (1996) 2778

[39] J. Dubois, et al., Phys. Rev. B 88 (2013) 085301.

[40] C. Grenier, et al., Phys. Rev. B 88 (2013) 085302.

[41] M. Moskalets, M. Büttiker, Phys. Rev. B 66 (2002) 205320.

[42] J. Keeling, A.V. Shytov, L.S. Levitov, Phys. Rev. Lett. 101 (2008) 196404.

[43] P. Hofer, C. Flindt, Phys. Rev. B 90 (2014) 235416. 